\title{
Fault-Tolerant Routing in Mesh-Connected 2D Tori
}

\author{
Eunseuk $\mathrm{Oh}^{1}$, Jong-Seok Kim ${ }^{2}$, and Hyeong-Ok Lee ${ }^{3}$ \\ 1 Department of Computer Science \\ Texas A\&M University, College Station, TX 77843-3112 \\ eunseuko@cs . tamu . edu \\ 2 Department of Computer Science \\ 3 Department of Computer Education \\ Sunchon National University, Sunchon, Chonnam, 540-742, Korea \\ \{rockhee, oklee\}@sunchon.ac.kr
}

\begin{abstract}
In this paper, we study natural conditions for $2 \mathrm{D}$ tori with a large number of faulty nodes to remain connected. Under the suggested connectivity conditions, we develop efficient routing algorithms in $2 \mathrm{D}$ tori with a large number of faulty nodes. As long as a given input torus and the meshes within the torus satisfy the conditions, the routing algorithm successfully constructs a fault-free path by using only local information of the network. Also, our algorithms do not require faulty nodes and faulty blocks to be a special structure such as convex, rectangle, while each mesh in a given torus can sustain as many faulty nodes as possible, provided that non-faulty nodes of the mesh are connected and the mesh holds the connectivity conditions. Specifically, for a torus sustaining up to $22.2 \%$ faulty nodes, in linear time, our algorithm constructs a faultfree path of length bounded by six times the shortest path length between the two nodes.
\end{abstract}

\section{Introduction}

Due to rapid progress in hardware technology, designing a distributed parallel computing system connecting a large number of multiprocessors has become feasible. In such a system, multiprocessors communicate by message passing. As the size of the network interconnecting multiprocessors continuously increases, routing in networks with a large number of faults becomes unavoidable. Let $G$ be a regular network of degree $n$. The node-connectivity of $G$ is the minimum integer $k$ such that removing any $k$ nodes in $G$ results in a disconnected or trivial graph. It is well-known that the node-connectivity of an $n$-regular graph is $n$. Thus, many fault-tolerant communication algorithms for $n$-regular graphs allow to contain up to $n-1$ faulty nodes. On the other hand, there has been much effort to try to allow $n$-regular networks to have more than $n-1$ faulty nodes [9 101011$]$.

Recently, a new connectivity measure, local subcube-connectivity has been introduced to identify conditions where hypercube networks with a very large 
number of faulty nodes still remain connected [34. A network is functional as long as every pair of non-faulty nodes in a network containing faulty nodes is still able to communicate. That is, any pair of two non-faulty nodes are connected by a fault-free path in such a network. Under the conditions that a hypercube network is still connected, Chen, et al. were able to contain up to $37.5 \%$ faulty nodes in hypercubes. Also, a formal theoretical analysis and experimental simulations have been provided to demonstrate a realistic and practical view of their connectivity measure 23,5 . They mentioned that their new connectivity measure is applicable to other hierarchical networks. In this paper, we establish the result for the applicability of their definitions to networks with a bounded degree such as tori and mesh.

A 2D torus is one of the most popular graph models used to represent interconnection networks [7]. A 2D torus represents parallel computers in which processors are connected by a two dimensional grid of communication links with wraparound connections. Due to a constant degree of tori and meshes, developing routing algorithms that can only tolerate constant faulty nodes would not be practical. In many real situations, fault-free routing paths in a torus or mesh can be constructed even though some nodes are disconnected from the network. Many faulty models on meshes that can contain faulty nodes greater than its tolerance has been introduced [1,6,12. Under their faulty models, a fault-free path can be constructed on the mesh containing a large number of faulty nodes. However, most faulty models require that faulty blocks form special structures such as rectangle, convex, or nonconvex (L, $\mathrm{T}$, or + shape), which regard all non-faulty nodes within these blocks as faulty. Our algorithms do not put such a restriction on faulty structures, and only assume that non-faulty nodes in the network make a connected graph and limit the number of faulty nodes on each mesh's boundary to be less than half.

For a given torus, our routing algorithm constructs a path, tracing meshes of similar sizes in the torus. As long as a given input torus and meshes within the torus satisfy the required conditions, the suggested algorithm successfully constructs the fault-free path even though the torus contains a large number of faulty nodes. Also, most of algorithms developed under faulty block models focused on wormhole routing, whose time complexity is more likely independent of the distance of the communication path if the message is long enough [8]. On the other hand, we consider our algorithm under the store-and-forward model, whose time complexity mostly depends on the distance of the communication path. Specifically, for a torus sustaining up to $22.2 \%$ faulty nodes, in linear time, our algorithm can construct a fault-free path of length bounded by six times the shortest path length between the two nodes. Further, our algorithm does not require global information about the network, and a fault-free path is constructed by only using information about directly connected nodes. Most of our discussions were focused on tori and their connectivity, but our results can easily be extended to meshes. 


\section{Preliminaries}

A two-dimensional torus $T$ (shortly, 2D torus or $n \times n$ torus) is an undirected graph with $n^{2}$ nodes whose label is identified by two coordinates $(i, j)$ and with $2 n^{2}$ edges. For a node $(i, j)$ in a torus, it has four neighbors $(i-1, j),(i+1, j)$, $(i, j-1)$, and $(i, j+1)$, where the "+" and "-" operations on indices are performed as modulo arithmetic. The distance between two nodes $u=\left(i_{1}, j_{1}\right)$ and $v=$ $\left(i_{2}, j_{2}\right)$ in $T$ is the shortest path length between them defined as

$$
\operatorname{dist}(u, v)=\min \left(\left|i_{1}-i_{2}\right|, n-\left|i_{1}-i_{2}\right|\right)+\min \left(\left|j_{1}-j_{2}\right|, n-\left|j_{1}-j_{2}\right|\right) .
$$

There are four simple and deterministic routing algorithms that connect the source node $u$ and the destination node $v$ : (1) the message is routed along the $X$ dimension and then along the $Y$ dimension, (2) the message is routed along the $X$ dimension and then along the $Y$ dimension using wraparound, (3) the message is routed along the $X$ dimension using wraparound and then along the $Y$ dimension, and (4) the message is routed along the $X$ dimension using wraparound and then the $Y$ dimension using wraparound. A minimal path is determined among them. The diameter, the maximum distance between any two nodes of an $n \times n$ torus, is $2\left\lfloor\frac{n}{2}\right\rfloor$.

\subsection{Balanced 4-ary Partitioning Tree}

A two-dimensional torus $T$ can be divided into meshes in many different ways. To explain uniformly, we assume that $T$ is divided into meshes of size at most $h^{2}$, where $h=\left\lceil\frac{n}{2^{\alpha}}\right\rceil$, and $\alpha$ is a natural number. Each node in a 2D mesh is labeled as $(i, j)$ and $0 \leq i, j<n$. The lower-most West node is labeled as $(0,0)$, and the upper-most East node is labeled as $(n-1, n-1)$. We first propose a labeling scheme to identify meshes in a torus when a torus is divided into meshes of similar sizes. The coordinates of the lower-most West nodes of the meshes are obtained by a balanced 4-ary partitioning tree which is defined below.

Definition 1. A balanced 4-ary partitioning tree is constructed as follows.

1. the root node is labeled as $n \times n$.

2. for each node with a label $h_{1} \times h_{2}$, generate the first, second, third, and fourth children of the node and label them as $h_{11} \times h_{21}, h_{12} \times h_{21}, h_{11} \times h_{22}$, and $h_{12} \times h_{22}$, respectively, where $h_{11}=\left\lfloor\frac{h_{1}}{2}\right\rfloor, h_{12}=\left\lceil\frac{h_{1}}{2}\right\rceil, h_{21}=\left\lfloor\frac{h_{2}}{2}\right\rfloor$, and $h_{22}=\left\lceil\frac{h_{2}}{2}\right\rceil$.

For the balanced 4-ary partitioning tree with depth $d$, there are $4^{d}$ leaf nodes. The depth of the root is 0 . Thus, an $n \times n$ torus is divided into $4^{d}$ meshes of size at most $h^{2}$, where $h=\left\lceil\frac{n}{2 d}\right\rceil$. The balanced 4-ary partitioning tree whose root is labeled as $13 \times 13$ is shown in Fig. 1. We assume that each node has pointers to nodes corresponding to neighboring meshes. The actual coordinates of the lower-most West node of a mesh in a torus can be calculated as follows. Let $\beta=\left\lfloor\frac{n}{2^{d}}\right\rfloor$. 


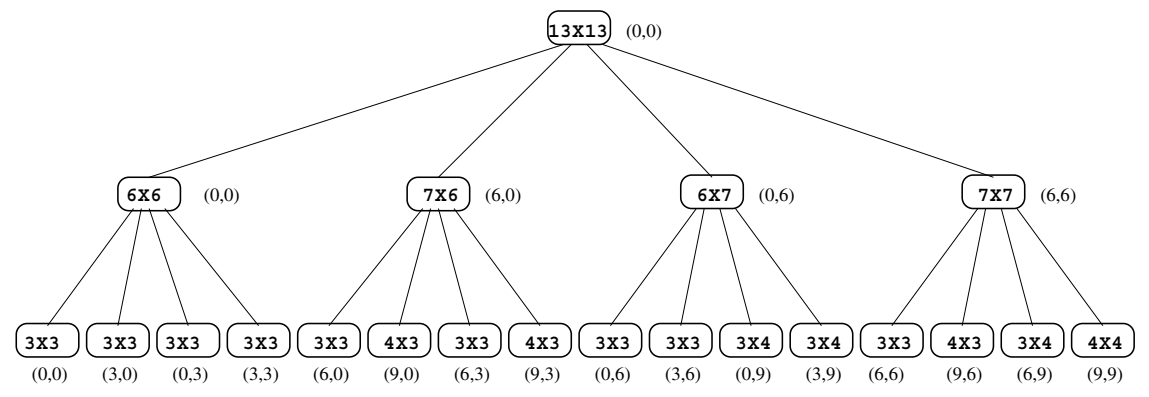

Fig. 1. Partition of a $13 \times 13$ torus by the balanced 4 -ary partitioning tree with depth 2

Coordinates for Lower-Most West Nodes of Meshes

1. the lower-most West node of a mesh identified by the root node is $(0,0)$.

2. for the lower-most West node $(i, j)$ in a mesh identified by the node in a balanced 4-ary partitioning tree at depth $d$, the lower-most West nodes of four meshes corresponding to the first, second, third, and fourth children of the node $(i, j)$ are $(i, j),(i+\beta, j),(i, j+\beta),(i+$ $\beta, j+\beta)$.

Now, mesh labels in a torus are obtained through its respective coordinates, $[i, j]$ where a mesh can be viewed as a node in a two dimensional grid. For any two meshes in a torus divided by the balanced 4-ary partitioning tree, the number of nodes on two adjoining boundaries are the same.

\section{The Local Mesh-Connectivity}

After applying the balanced 4-ary partitioning tree with depth $d$ to an $n \times n$ torus, a label $[i, j]$ corresponds to a mesh whose coordinate of the lower-most West node is $(\beta \cdot i, \beta \cdot j)$, where $\beta=\left\lfloor\frac{n}{2^{d}}\right\rfloor$. The size of the mesh is decided according to the definition of a balanced 4-ary partitioning tree. The set of meshes obtained by applying the balanced 4-ary partitioning tree with the depth $d$ is called a $d$ mesh set, and a mesh in the $d$-mesh-set is called $d$-mesh. In addition, the distance between the two $d$-meshes $M_{1}=\left[i_{1}, j_{1}\right]$ and $M_{2}=\left[i_{2}, j_{2}\right]$ is called $d$-distance and defined as

$$
\Delta\left(M_{1}, M_{2}\right)=\min \left(\left|i_{1}-i_{2}\right|, 2^{d}-\left|i_{1}-i_{2}\right|\right)+\min \left(\left|j_{1}-j_{2}\right|, 2^{d}-\left|j_{1}-j_{2}\right|\right) .
$$

Definition 2. The 2D torus $T$ is locally $d$-mesh-connected if in each $d$-mesh in $T$, the non-faulty nodes in the $d$-mesh are connected.

For any given two non-faulty nodes $u$ and $v$ in a locally $d$-mesh-connected torus, a sufficient condition to exist a fault-free path between them is provided below. We denote it as $u \stackrel{p}{\sim} v$ if there is a path $p$ between $u$ and $v$, and as $u \rightarrow v$ if $u$ is directly connected to $v$. 
Theorem 1. If, on any side of a boundary of size $h$ in each d-mesh, the number of faulty nodes is at most $\left\lfloor\frac{h-1}{2}\right\rfloor$, then the non-faulty nodes in a locally d-meshconnected $2 D$ torus $T$ make a connected graph.

Proof. To prove that the non-faulty nodes in a locally $d$-mesh-connected 2D torus make a connected graph, it suffices to show that for any two given nodes $u$ and $v$ in a $2 \mathrm{D}$ torus, there is a fault-free path $u \sim v$. Since the non-faulty nodes in a $d$-mesh in $T$ are connected, if $u$ and $v$ is in a $d$-mesh $M$, then $u \stackrel{p}{\sim} v$ where all nodes on $p$ are in $M$. Suppose the node $u$ is in a $d$-mesh $M$ and the node $v$ is in another $d$-mesh $M^{\prime}$. Let a neighboring $d$-mesh of $M$ be $M_{1}$. Then, there are $h$ pairs of adjacent nodes such that one is on the boundary of $M$ and another is on the boundary of $M_{1}$. Since we assume that on any boundary of a $d$-mesh, less than half of the nodes are faulty, there must be a path of adjacent non-faulty nodes $b_{0} \rightarrow b_{1}$ such that $b_{0}$ is in $M$ and $b_{1}$ is in $M_{1}$. By the definition of $d$-mesh connectivity, we have $u \stackrel{p}{\sim} b_{0}$, where all nodes on $p$ are in $M$. Thus, we can construct a fault-free path $u \stackrel{p}{\sim} b_{0} \rightarrow b_{1}$. We continue this construction until we have $u \sim v^{\prime}$ such that $v^{\prime}$ is a non-faulty node on the boundary of $M^{\prime}$. Inductively, the fault-free path $u \sim v^{\prime}$ can be constructed by extending the path between them going through meshes between $M$ and $M^{\prime}$. Since non-faulty nodes in $M^{\prime}$ are connected, there is a fault-free path $v^{\prime} \stackrel{p^{\prime}}{\sim} v$ such that all nodes on $p^{\prime}$ are in $M^{\prime}$. Therefore, the concatenation of the path $u \sim v^{\prime}$ and the path between $v^{\prime} \sim v$ gives a fault-free path $u \sim v$.

Under the above boundary condition, the non-faulty nodes in a locally $d$ mesh-connected 2D torus $T$ make a connected graph, though $T$ may contain a very large number of faulty nodes. It shows that the local $d$-mesh connectivity leads to the global connectivity of the network with a large number of faulty nodes as long as the boundary condition is satisfied. Furthermore, as pointed out in [3], the local mesh-connectivity can also be detected in a distributed way by using a local managing program on a processor in each mesh.

Suppose that a $d$-mesh contains faulty nodes up to less than half of its total number of nodes. When $d$ is small enough, the number of faulty nodes that can be contained in a locally $d$-mesh-connected torus $T$ would be close to half of the total number of nodes in $T$. To give an intuition of the number of faulty nodes, consider a square $n \times n$ torus and set $d=\log \frac{n}{h}$. Since a $d$-mesh has size of $h^{2}$ and $h=\frac{n}{2^{d}}$, a $d$-mesh may contain up to $4^{d}\left(\frac{1}{2}\left(\frac{n}{2^{d}}\right)^{2}-1\right)=\frac{n^{2}}{2}-4^{d}$ faulty nodes. According to the sufficient condition on the boundary of a mesh, a $\log \frac{n}{3}$-mesh can contain up to 2 faulty nodes.

Corollary 1. Under the boundary condition, if each d-mesh in a locally d-mesh connected torus $T$ has at most 2 faulty nodes, then the non-faulty nodes in $T$ make a connected graph, where $d=\log \frac{n}{3}$.

Corollary 2. Under the boundary condition, a locally d-mesh-connected torus $T$ is also locally $k$-mesh-connected for all $k<d$. 
So far, we assume that $d$ is fixed in a locally $d$-mesh-connected torus and its non-faulty nodes are connected as long as the number of faulty nodes in any side of a boundary of $d$-mesh is less than half of the nodes on the boundary. We generalize the previous condition to allow a faulty $d$-mesh(the mesh whose nodes are all faulty), while the torus remains connected.

Definition 3. The 2D torus $T$ is locally mesh-connected if each $d$-mesh, $d \geq 1$, in $T$ is contained in an $h$-mesh whose non-faulty nodes are connected, $h \leq d$.

Let $\delta$ be the largest integer such that the locally mesh-connected 2D torus $T$ is locally $\delta$-mesh-connected. Such a number $\delta$ always exists. For example, $\delta=0$. In this case, the locally mesh-connected 2D torus itself is locally 0-meshconnected. If the boundary condition is satisfied, all non-faulty nodes in $T$ are connected. The following theorem directly directly from the definition of $\delta$.

Theorem 2. If, on any side of a boundary of size $h$ in each $\delta$-mesh, the number of faulty nodes is at most $\left\lfloor\frac{h-1}{2}\right\rfloor$, then the non-faulty nodes in a locally meshconnected $2 D$ torus make a connected graph.

\section{Routing in Locally Mesh-Connected Tori}

As described in Theorem 1 a path between two given non-faulty nodes in a locally mesh-connected torus can be constructed using only non-faulty nodes when all $d$-mesh satisfy the boundary condition. Before we present routing algorithms in a locally mesh-connected torus, we discuss the worst case to find two non-faulty nodes $u$ and $u_{1}$, where $u$ is in a mesh $M$ and $u_{1}$ is in the adjoining boundary of a mesh $M_{1}$. Without loss of generality, we assume that the size of $M$ is $h_{1} h_{2}$ and the size of $M_{1}$ is $h_{3} h_{2}$. The worst case would take time $O\left(h_{1} h_{2}\right)$. We will focus more on routing algorithms whose time complexity is linear and the length of path. An example case that takes roughly half of the size of a $d$-mesh is given in Fig. 2 .

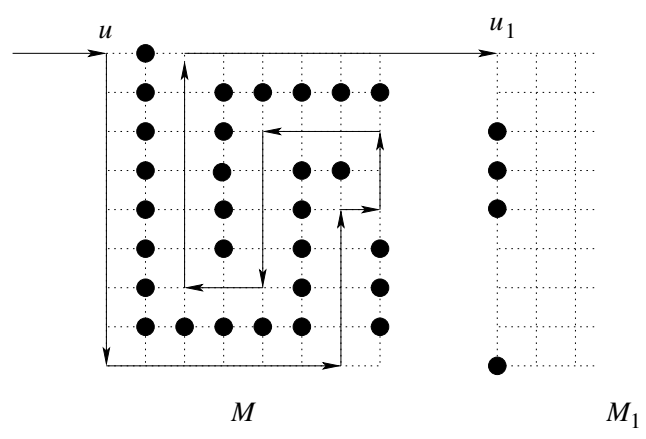

Fig. 2. An example for connecting two non-faulty nodes, one is in $M$ and the other is in $M_{1}$ 


\subsection{Routing in a Locally $d$-Mesh-Connected Torus}

Assume that a $d$-mesh $M$ contains the source node $u$ and another $d$-mesh $M^{\prime}$ contains the destination node $v$. An efficient routing algorithm for a locally $d$ mesh-connected torus is given in Fig. 3 .

\section{Routing}

Input: an $n \times n$ torus $T$ and non-faulty nodes $u$ and $v$ in it

Output: a fault-free path $P=u \sim v$ or return FAIL if $T$ is not locally $d$-mesh-connected or it does not satisfy the boundary condition.

\{Assume that $u$ is in a $d$-mesh $M$ and $v$ is in another $d$-mesh $M^{\prime}$.

Phase 1: find labels for $d$-meshes connecting $M$ and $M^{\prime}$ whose $d$-distance is $\Delta\left(M, M^{\prime}\right)$.

Let such $d$-meshes be $M\left(=M_{0}\right), M_{1}, \ldots, M^{\prime}=\left(M_{\Delta\left(M, M^{\prime}\right)}\right)$;

Phase 2: find a fault-free path from $u$ in $M$ to a non-faulty node in $M^{\prime}$;

2.2 initialize the path $P=u$, and let $w=u$;

2.3 for $k=0$ to $\Delta\left(M, M^{\prime}\right)-1$ do

find a fault-free path $b_{1} \rightarrow b_{2}$ such that $b_{1}$ is in $M_{k}$ and $b_{2}$ is in $M_{k+1}$;

extend the path $P$ to $w \stackrel{p}{\sim} b_{1} \rightarrow b_{2}$, where all nodes of $p$ are in $M_{k}$; let $w=b_{2}$;

Phase 3: find a fault-free path from a non-faulty node to $v$ in $M^{\prime}$;

\{at this point, $w$ is on a boundary of $M^{\prime}$. \}

extend the path $P$ to $w \stackrel{p^{\prime}}{\sim} v$, where all nodes of $p^{\prime}$ are in $M^{\prime}$;

if, at any point, the algorithm can not proceed, return FAIL;

Fig. 3. Routing in a locally $d$-mesh-connected torus

Theorem 3. For two given non-faulty nodes $u$ in a $d$-mesh $M$ and $v$ in another d-mesh $M^{\prime}$ in a locally d-mesh-connected torus, the algorithm Routing constructs a fault-free path $u \sim v$ in time $O(h n)$ if all d-meshes satisfy the boundary condition, where $h=\left\lceil\frac{n}{2^{d}}\right\rceil$.

Proof. The construction of a fault-free path $P$ starts from the node $u$ and traverses $d$-meshes detected in Phase 1. When $u$ is in $M$ and $v$ is in $M^{\prime}, d$ meshes connecting $M$ and $M^{\prime}$ whose length is $\Delta\left(M, M^{\prime}\right)$ can be found in time $2 \cdot 2^{d}=O\left(\frac{n}{h}\right)$, which is chosen to have the minimum number of $d$-meshes among four possible routes from the label of $M$ to the label of $M^{\prime}$ with or without wraparound. In the $k$ th execution of the loop in Phase 2 , a non-faulty node $u$ in $M_{k}$ is connected to a non-faulty node $b_{2}$ in $M_{k+1}$ going through $b_{1}$ such that $b_{1} \rightarrow b_{2}$, where $b_{1}$ is on a boundary of $M_{k}$ and $b_{2}$ is on a boundary of $M_{k+1}$. Finding the path $b_{1} \rightarrow b_{2}$ takes time $O(h)$. Since a non-faulty node $u$ can be connected to these adjacent nodes in time $O\left(h^{2}\right)$, we can find a fault-free path $u \sim b_{2}$ in time $O\left(h^{2}\right)$. After $\Delta\left(M, M^{\prime}\right)$ executions of the loop in Phase 2, the 
node $w$ is in a boundary of $M^{\prime}$. Since the non-faulty nodes in $M^{\prime}$ make a connected graph, in time $O\left(h^{2}\right)$, we can find a fault-free path $w \sim v$, and can extend the path $P$ to $w \sim v$. Therefore, if the $n \times n$ torus is locally $d$-mesh-connected and all $d$-meshes satisfy the boundary condition, then the algorithm Routing constructs a fault-free path $u \sim v$. The running time of the algorithm is bounded by $O\left(\frac{n}{h}+\Delta\left(M, M^{\prime}\right) h^{2}\right)=O(h n)$, since $\Delta\left(M, M^{\prime}\right)=O\left(\frac{n}{h}\right)$.

In the case where $h$ is a small constant, for example $h \leq 4$, the algorithm Routing runs in linear time. As shown in Fig. 2] some structures of a $d$-mesh take time $O\left(h^{2}\right)$ to search non-faulty nodes. Thus, our time complexity is reasonably tight even for large $h$. The length of the path $P$ constructed by Routing is bounded by $\left(\Delta\left(M, M^{\prime}\right)+1\right) h^{2}-1$ because the length of $P$ is increased by at most $h^{2}$ in each execution of the loop in Phase 2 and the length of the path constructed in Phase 3 is bound by $h^{2}-1$. Again, if $h$ is small, the length of the path $P$ becomes $O(n)$. Specifically, for a torus divided into $3 \times 3$ meshes by a 4 -ary balanced partitioning tree, a locally $d$-mesh-connected torus may contain up to $\frac{2}{9} n^{2}$ faulty nodes, which is $22.2 \%$ of the total number of nodes in a torus. The number of nodes that can be contained in a locally mesh-connected torus would increase as the size of a $d$-mesh increases at the cost of the path length. For a locally $d$-mesh-connected torus consisting of $3 \times 3$ meshes, we have the following corollary.

Corollary 3. For a locally d-mesh-connected torus consisting of $\log \frac{n}{3}$-meshes, in linear time, the algorithm Routing constructs a fault-free path of length bounded by $6 \Delta\left(M, M^{\prime}\right)$.

\subsection{Routing in a Locally Mesh-Connected Torus}

Similarly a fault-free path $u \sim v$ in a locally mesh-connected torus $T$ can be constructed when all $\delta$-meshes satisfy the boundary condition. A routing algorithm for $T$ is given in Fig. 4. The largest integer $\delta$ such that $T$ is locally $\delta$-mesh connected is not given in our algorithm. If the value $\delta$ is known, a path $u \sim v$ can be constructed by the algorithm Routing.

Theorem 4. If the input torus $T$ of the algorithm $\delta$-Routing is locally meshconnected, then for two non-faulty nodes $u$ and $v$, and $\delta$ such that $T$ is locally $\delta$-mesh-connected, the algorithm $\delta$-Routing constructs a fault-free path $u \sim v$ in time $O(h n)$, provided that all $\delta$-meshes satisfy the boundary condition, where $h=\left\lceil\frac{n}{2^{\delta}}\right\rceil$.

Proof. Suppose a path $P=u \sim w, w \neq v$ is constructed inductively. By the definition of the value $\delta$, we must have an integer $k \geq \delta$ such that $w$ is in a $k$-mesh $M$ whose non-faulty nodes are connected, and a fault-free path $b_{1} \rightarrow b_{2}$ can be constructed between $b_{1}$ in a boundary of $M$ and $b_{2}$ in a boundary of $k$-mesh $M_{1}$. However, since we do not know the value $\delta$, we will try all possible values from $k=\left\lceil\log \frac{n}{2}\right\rceil$ to find a fault-free path $b_{1} \rightarrow b_{2}$. In the worst case, $k=\delta$, we have $w \stackrel{p}{\sim} b_{1} \rightarrow b_{2}$, where all nodes of $p$ are in a $\delta$-mesh. Using the balanced 4-ary 


\section{$\delta$-Routing}

Input: an $n \times n$ torus $T$ and non-faulty nodes $u$ and $v$ in $T$

Output: a fault-free path $P=u \sim v$ or return FAIL if $T$ is not locally mesh-connected or it does not satisfy the boundary condition.

1. let $w=u$;

2. initialize the path $P=w$;

3. while $w \neq v$ do $k=\left\lceil\log \frac{n}{2}\right\rceil ;$ done $=$ false;

while done=false and $w$ is not in $M^{\prime}$ do

$3.1 \quad$ find a $k$-mesh $M_{0}$ with $w$ and its neighboring $k$-mesh $M_{1}$ such that $\Delta\left(M_{1}, M^{\prime}\right)=\Delta\left(M_{0}, M^{\prime}\right)-1$, where $M^{\prime}$ is a $k$-mesh with $v$;

3.2 if there is $w \stackrel{p}{\sim} b_{1} \rightarrow b_{2}$ such that $b_{1}$ is in $M_{0}$ and $b_{2}$ is in $M_{1}$; then

extend the path $P$ to the path $w \stackrel{p}{\sim} b_{1} \rightarrow b_{2}$, where all nodes of $p$ are in $M_{0}$; let $w=b_{2}$;

else done $=$ true;

3.3 if $w$ is in $M^{\prime}$

$$
k=k-1
$$

while there is no fault-free unused path $w \sim v$ do

$$
k=k-1 \text {; }
$$

let $M^{\prime \prime}$ be a $k$-mesh with $v$;

extend the path $P$ to $w \stackrel{p^{\prime}}{\sim} v$, where all nodes of $p^{\prime}$ are in $M^{\prime \prime}$;

return $P=u \sim v$;

if, at any point, the algorithm can not proceed, return FAIL;

Fig. 4. Routing in a locally mesh-connected torus

partitioning tree, Step 3.1 of the algorithm $\delta$-Routing can be done in a constant time after identifying a $k$-mesh $M_{0}$ with $u$, which takes $O\left(\log \frac{n}{2}\right)$. Since we need to check whether non-faulty nodes in a $k$-mesh are connected and it satisfies the boundary condition for each value $k$, Step 3.2 of the algorithm $\delta$-Routing takes $O\left(2^{2}\right)+O\left(4^{2}\right)+\cdots+O\left(h^{2}\right)=O\left(h^{2}\right)$. Thus, the total time of Step 3.1 and Step 3.2 is bounded by $\log \frac{n}{2}+h^{2} \Delta\left(M, M^{\prime}\right)=O(h n)$, where $h=\left\lceil\frac{n}{2^{\delta}}\right\rceil$. If $w$ is in a $k$-mesh $M^{\prime}$, then nodes $w$ and $v$ are in the same $\delta$-mesh. We try to find a fault-free path $w \sim v$ in the smallest size mesh(thus, the largest $k$ ) from the value $k$ found in Step 3.2. Thus, a fault-free path $w \sim v$ can be constructed in time $O\left(h^{2}\right)$. It shows that in time $O(h n)$, the algorithm $\delta$-Routing constructs a fault-free path $u \sim v$ in the locally mesh-connected torus if all $\delta$-meshes satisfy the boundary condition.

In general, the running time of the algorithm $\delta$-Routing would be much better than $O(h n)$. For example, if a torus $T$ contains relatively fewer faulty nodes so that most small meshes in $T$ satisfy the boundary condition and nonfaulty nodes in them are connected, then the largest value $k$ can be found in the earlier iteration of Step 3.2. Thus, the path $P$ can be extended in meshes smaller than $h^{2}$ in most cases. 


\section{Conclusion}

We have studied the connectivity measure for a 2D torus with a large number of faulty nodes to remain connected, and established the result for tori. Our study shows that the local subgraph-connectivity suggested in 3,4] also successfully extends to networks with a bounded degree. The algorithm developed for tori meeting these conditions is practical and efficient in most cases. Further, our algorithm does not require global information about the network, and a fault-free path is constructed by using only information about directly connected nodes. In this paper, we only consider the routing problem. Other communication problems in tori such as broadcasting and gossiping would be interesting to study under this new connectivity measure. In addition, our result can easily be extended to meshes. Without considering wraparound connections, a 2D torus has the same structure as a $2 \mathrm{D}$ mesh. Thus, our algorithm can be applied to a $2 \mathrm{D}$ mesh with a slight modification.

\section{References}

1. S. Chalasani and R.V. Boppana, "Communication in Multicomputers with Nonconvex faults," Proc. Int'l Symp. Computer Architecture, pp. 268-277, 1990.

2. J. Chen, I. A. Kanj, and G. Wang, "Hypercube Network Fault Tolerance: A Probabilistic Approach," Proc. Int'l Conf. Parallel Processing, pp. 65-72, 2002.

3. J. Chen, G. Wang, and S. Chen, "Locally Subcube-Connected Hypercube Networks: Theoretical Analysis and Experimental Results," IEEE Trans. Computers, vol. 51, no. 5, pp. 530-540, 2002.

4. J. Chen, G. Wang, and S. Chen, "Routing in Hypercube Networks with A Constant Fraction of Faulty Nodes," Journal of Interconnection Networks, vol. 2, pp. 283294, 2001.

5. J. Chen, T. Wang, and E. Oh, "Adaptive Routing: Centralized versus Distributed," Technical Report, Texas A\&M University, 2002.

6. A.A. Chien and J.H. Kim, "Planar-Adaptive Routing: Low-Cost Adaptive Networks for Multiprocessors," Proc. Int'l Symp. Computer Architecture, pp. 268-277, 1990.

7. W.J. Dally and C.L. Seitz, "The torus Routing Chip," Distributed Computing, pp. 187-196, 1986.

8. W.J. Dally and C.L. Seitz, "Deadlock-free message routing in multiprocessor interconnection network," IEEE Trans Comput. vol. 36, no. 5, pp. 547-553, 1987.

9. Q.-P. Gu and S. Peng, " $k$-Pairwise Cluster Fault Tolerant Routing in Hypercubes," IEEE Trans. Computers, vol. 46, pp. 1042-1049, 1997.

10. Q.-P. Gu and S. Peng, "Unicast in Hypercubes with Large Number of Faulty Nodes," IEEE Trans. Parallel and Distributed Systems, vol. 10, pp. 946-975, 1999.

11. S. Latifi, M. Hedge, and M. Naraghi-Pour, "Conditional Connectivity Measures for Large Multiprocessor Systems," IEEE Trans. Computers, vol. 43, pp. 218-222, 1994.

12. C.C. Su and K.G. Shin, "Adaptive Fault-Tolerant Deadlock-Free Routing in Meshes and Hypercubes," IEEE Trans. Computers, vol. 45, no. 6, pp. 666-683, 1996. 